\title{
Erratum To: Virtual house calls for Parkinson disease (Connect.Parkinson): study protocol for a randomized, controlled trial
}

\author{
Meredith A. Achey, Christopher A. Beck, Denise B. Beran, Cynthia M. Boyd*, Peter N. Schmidt, Allison W. Willis, \\ Sara S. Riggare, Richard B. Simone, Kevin M. Biglan and E. Ray Dorsey
}

After the publication of this article [1], it was discovered that eleven of the trials listed in the original article's Table 1 [1], had been erroneously identified as taking place in the home [2-12]. These studies actually evaluated physician videoconferencing visits with patients located in clinics. To ensure accuracy, we repeated the literature search in September of 2015, using the same search terms reported in the article and filtered for a publication date prior to July 1, 2014 (the original work was performed in June 2014.) We searched PubMed using the terms 'telemedicine AND home AND randomized' (378 results), 'randomized AND video AND home' (259 results), 'videoconferencing AND randomized' (178 results), and 'virtual AND visits AND home' (33 results), and reviewed the 141 studies identified in the review by Dr. Wootton mentioned in the article [13]. Of the 848 search results and 141 studies identified by Dr. Wootton, a total of six randomized controlled trials involving physician video calls directly to a patient in the home were identified (four from the original review [14-17] and two additional studies $[18,19]$ identified through the new search). The eleven misidentified articles have been removed from the Corrected Table 1, and included for clarity as Erratum Table 2. The final paper listed in Erratum Table 2, Bishop JE et al. [3], has also been corrected here: our article reported 19 subjects, but the abstract indicates that 17 completed the study. We sincerely apologize for the oversight and any inconvenience these errors might have caused.

\footnotetext{
*Correspondence: cyboyd@jhmi.edu

Division of Geriatric Medicine and Gerontology, Department of Medicine, Johns Hopkins University School of Medicine, 5200 Eastern Avenue, MFL 7th Floor, Center Tower, Baltimore, MD 21224, USA
} 
Corrected Table 1 Randomized, controlled trials involving video based virtual house calls from physicians $(N=6)$

\begin{tabular}{|c|c|c|c|c|c|c|c|}
\hline Study & Year & Sample size & Study population & Intervention(s) & Duration & Primary outcomes & Results \\
\hline \multirow{2}{*}{$\begin{array}{l}\text { Dorsey ER } \\
\text { et al. [14] }\end{array}$} & \multirow[t]{2}{*}{2013} & \multirow[t]{2}{*}{20} & \multirow{2}{*}{$\begin{array}{l}\text { Individuals with } \\
\text { Parkinson disease }\end{array}$} & \multirow{2}{*}{$\begin{array}{l}\text { Randomized to (1) in-person care } \\
\text { or (2) care via telemedicine }\end{array}$} & \multirow[t]{2}{*}{7 months } & - Feasibility & - Virtual house calls were feasible \\
\hline & & & & & & - Quality of life & - As effective as in-person care \\
\hline \multirow[t]{2}{*}{$\begin{array}{l}\text { McCrossan B } \\
\text { et al. [15] }\end{array}$} & \multirow[t]{2}{*}{2012} & \multirow[t]{2}{*}{83} & \multirow[t]{2}{*}{$\begin{array}{l}\text { Infants with congenital } \\
\text { heart defects }\end{array}$} & \multirow{2}{*}{$\begin{array}{l}\text { Randomized to (1) videoconferencing } \\
\text { support, (2) telephone support, } \\
\text { or (3) control }\end{array}$} & \multirow[t]{2}{*}{10 weeks } & - Acceptability & $\begin{array}{l}\text { - Clinicians were more confident in treating } \\
\text { patients in video visits vs. telephone }\end{array}$ \\
\hline & & & & & & - Healthcare resource utilization & $\begin{array}{l}\text { - Parents were satisfied with video visits } \\
\text { - Healthcare resource utilization was } \\
\text { lower in video-conferencing group }\end{array}$ \\
\hline \multirow{4}{*}{$\begin{array}{l}\text { Leon A } \\
\text { et al. [17] }\end{array}$} & \multirow[t]{4}{*}{2011} & \multirow[t]{4}{*}{83} & \multirow[t]{4}{*}{ Individuals with HIV } & \multirow{4}{*}{$\begin{array}{l}\text { Randomized to (1) usual care or (2) Virtual } \\
\text { Hospital care for one year, then crossed } \\
\text { over after one year }\end{array}$} & \multirow[t]{4}{*}{2 years } & - Clinical & - Satisfaction with Virtual Hospital was high \\
\hline & & & & & & - Healthcare resource utilization & $\begin{array}{l}\text { - Clinical outcomes were similar for } \\
\text { both groups }\end{array}$ \\
\hline & & & & & & - Quality of life & \\
\hline & & & & & & - Satisfaction & \\
\hline \multirow{3}{*}{$\begin{array}{l}\text { Morgan GJ } \\
\text { et al. [16] }\end{array}$} & \multirow[t]{3}{*}{2008} & \multirow[t]{3}{*}{30} & \multirow{3}{*}{$\begin{array}{l}\text { Parents of children with } \\
\text { severe congenital } \\
\text { heart disease }\end{array}$} & \multirow{3}{*}{$\begin{array}{l}\text { Randomized to (1) telephone or } \\
\text { (2) videoconferencing follow-up }\end{array}$} & \multirow[t]{3}{*}{6 weeks } & - Parents' anxiety & \multirow{3}{*}{$\begin{array}{l}\text { Videoconferencing decreased anxiety } \\
\text { levels compared to telephone and } \\
\text { allowed better clinical information }\end{array}$} \\
\hline & & & & & & - Clinical & \\
\hline & & & & & & - Clinician and patient satisfaction & \\
\hline \multirow[t]{2}{*}{$\begin{array}{l}\text { Dallolio L } \\
\text { et al. [19] }\end{array}$} & \multirow[t]{2}{*}{2008} & \multirow[t]{2}{*}{137} & \multirow[t]{2}{*}{$\begin{array}{l}\text { Individuals with } \\
\text { spinal cord injury }\end{array}$} & \multirow{2}{*}{$\begin{array}{l}\text { Randomized to (1) home (or nursing home } \\
\text { or hospital) telemedicine (physician and } \\
\text { nurse) and telerehabilitation (therapist) } \\
\text { or (2) standard post-discharge care }\end{array}$} & \multirow[t]{2}{*}{6 months } & - Clinical & $\begin{array}{l}\text { - Telemedicine patients at one out of four } \\
\text { sites had statistically significantly better } \\
\text { functional improvement }\end{array}$ \\
\hline & & & & & & - Satisfaction & $\begin{array}{l}\text { - Satisfaction with interactions with nursing } \\
\text { and medical staff and information and } \\
\text { treatment received were higher in the } \\
\text { telemedicine group }\end{array}$ \\
\hline \multirow[t]{4}{*}{$\begin{array}{l}\text { Whitlock WL } \\
\text { et al. [18] }\end{array}$} & \multirow[t]{4}{*}{2000} & \multirow[t]{4}{*}{28} & \multirow[t]{4}{*}{$\begin{array}{l}\text { Individuals with } \\
\text { Type II diabetes }\end{array}$} & \multirow[t]{4}{*}{$\begin{array}{l}\text { Randomized to (1) home videoconferencing } \\
\text { (monthly physician calls and weekly nurse } \\
\text { calls) or (2) standard in-person care }\end{array}$} & 3 months & - Clinical & $\begin{array}{l}\text { Some clinical outcomes improved } \\
\text { significantly more in the telemedicine } \\
\text { group }\end{array}$ \\
\hline & & & & & & - Quality of life & - Quality of life was unchanged \\
\hline & & & & & & - Satisfaction & $\begin{array}{l}\text { - Physicians and case managers reported } \\
\text { high subjective utility of telemedicine }\end{array}$ \\
\hline & & & & & & & - Technology problems were an obstacle \\
\hline
\end{tabular}


Erratum Table 2 Randomized, controlled trials involving video based physician visits with patients in clinical environments ( $N=11$ )

\begin{tabular}{|c|c|c|c|c|c|c|c|c|}
\hline Study & Year & Sample size & Study population & Intervention(s) & Duration & & rimary outcomes & Results \\
\hline Fortney JC et al. [8] & 2013 & 364 & Individuals with depression & $\begin{array}{l}\text { Randomized to practice-based or } \\
\text { telemedicine-base collaborative care }\end{array}$ & 18 months & $\cdot$ & Clinical & $\begin{array}{l}\text { Telemedicine-based collaborative } \\
\text { care yielded better outcomes for } \\
\text { depressed patients }\end{array}$ \\
\hline \multirow[t]{2}{*}{ Moreno FA et al. [9] } & \multirow[t]{2}{*}{2012} & \multirow[t]{2}{*}{167} & \multirow[t]{2}{*}{$\begin{array}{l}\text { Hispanic adults with } \\
\text { depression }\end{array}$} & \multirow{2}{*}{$\begin{array}{l}\text { Randomized to telemedicine care } \\
\text { from a psychiatrist or usual care } \\
\text { from a primary care physician }\end{array}$} & \multirow[t]{2}{*}{6 months } & $\cdot$ & Clinical & $\begin{array}{l}\text { All participants improved on clinical } \\
\text { measures }\end{array}$ \\
\hline & & & & & & $\cdot$ & Quality of life & $\begin{array}{l}\text { - Time to improvement was shorter in } \\
\text { telemedicine group }\end{array}$ \\
\hline \multirow[t]{2}{*}{ Ferrer-Roca O et al. [7] } & \multirow[t]{2}{*}{2010} & \multirow[t]{2}{*}{800} & \multirow[t]{2}{*}{$\begin{array}{l}\text { Primary care patients referred } \\
\text { for specialized care }\end{array}$} & \multirow[t]{2}{*}{$\begin{array}{l}\text { Randomized to face-to-face hospital } \\
\text { referral or telemedicine from specialist }\end{array}$} & \multirow[t]{2}{*}{6 months } & \multirow{2}{*}{\multicolumn{2}{|c|}{ - Quality of life }} & $\begin{array}{l}\text { - Telemedicine care was comparable to } \\
\text { face-to-face care }\end{array}$ \\
\hline & & & & & & & & $\begin{array}{l}\text { Diagnosis and examination to start } \\
\text { treatment were faster in the } \\
\text { telemedicine group }\end{array}$ \\
\hline \multirow[t]{2}{*}{ Stahl JE, Dixon RF [12] } & \multirow[t]{2}{*}{2010} & \multirow[t]{2}{*}{175} & \multirow[t]{2}{*}{$\begin{array}{l}\text { Patients in a general } \\
\text { primary care practice }\end{array}$} & \multirow[t]{2}{*}{$\begin{array}{l}\text { Interviewed face-to-face and via } \\
\text { videoconferencing, order randomized }\end{array}$} & \multirow[t]{2}{*}{2 visits } & $\cdot$ & Satisfaction & $\begin{array}{l}\text { - Patients and providers were highly } \\
\text { satisfied with videoconferencing but } \\
\text { preferred face-to-face }\end{array}$ \\
\hline & & & & & & $\cdot$ & Willingness to pay & $\begin{array}{l}\text { - Technical quality of video calls had } \\
\text { significant impact on satisfaction }\end{array}$ \\
\hline \multirow[t]{2}{*}{ Dorsey ER et al. [6] } & \multirow[t]{2}{*}{2010} & \multirow[t]{2}{*}{14} & \multirow{2}{*}{$\begin{array}{l}\text { Individuals with } \\
\text { Parkinson disease }\end{array}$} & \multirow{2}{*}{$\begin{array}{l}\text { Randomized to usual care } \\
\text { or care via telemedicine }\end{array}$} & \multirow[t]{2}{*}{6 months } & \multirow{2}{*}{\multicolumn{2}{|c|}{ - Feasibility }} & - Virtual house calls were feasible \\
\hline & & & & & & & & $\begin{array}{l}\text { Virtual house calls improved } \\
\text { disease-specific measures significantly } \\
\text { compared to usual care. }\end{array}$ \\
\hline \multirow[t]{2}{*}{ Dixon RF, Stahl JE [5] } & \multirow[t]{2}{*}{2009} & \multirow[t]{2}{*}{175} & \multirow[t]{2}{*}{$\begin{array}{l}\text { Patients in a general } \\
\text { primary care practice }\end{array}$} & \multirow{2}{*}{$\begin{array}{l}\text { Randomized to one virtual visit } \\
\text { and one face-to-face, or two } \\
\text { face-to-face consultations }\end{array}$} & \multirow[t]{2}{*}{2 visits } & $\cdot$ & Diagnostic agreement & $\begin{array}{l}\text { - Physicians and patients highly satisfied } \\
\text { with virtual visits }\end{array}$ \\
\hline & & & & & & · & Satisfaction & $\begin{array}{l}\text { Diagnostic agreement between } \\
\text { virtual and in-person evaluation } \\
\text { was similar to comparison of } \\
\text { two in-person evaluations }\end{array}$ \\
\hline \multirow[t]{3}{*}{ Ahmed SN et al. [2] } & \multirow[t]{3}{*}{2008} & \multirow[t]{3}{*}{41} & \multirow[t]{3}{*}{ Epilepsy patients } & $\begin{array}{l}\text { Randomized to telemedicine } \\
\text { follow up or conventional }\end{array}$ & 1 visit & $\cdot$ & Cost effectiveness & $\begin{array}{l}\text { - } 90 \% \text { of patients in both groups } \\
\text { satisfied with quality of services }\end{array}$ \\
\hline & & & & & & & $\begin{array}{l}\text { Cost to patients } \\
\text { and caregivers }\end{array}$ & $\begin{array}{l}\text { - Cost of telemedicine production was } \\
\text { similar to patient savings }\end{array}$ \\
\hline & & & & & & · & Satisfaction & \\
\hline O'Reilly R et al. [10] & 2007 & 495 & $\begin{array}{l}\text { Patients referred for } \\
\text { psychiatric consult }\end{array}$ & $\begin{array}{l}\text { Randomized to face to face } \\
\text { or telepsychiatry }\end{array}$ & 4 months & • & Clinical & $\begin{array}{l}\text { - Similar outcomes were seen } \\
\text { in both arms }\end{array}$ \\
\hline & & & & & & $\cdot$ & Cost effectiveness & $\begin{array}{l}\text { - Telepsychiatry was at least } 10 \% \text { less } \\
\text { expensive than in-person care }\end{array}$ \\
\hline & & & & & & • & Satisfaction & - Both groups expressed similar satisfaction \\
\hline
\end{tabular}


Erratum Table 2 Randomized, controlled trials involving video based physician visits with patients in clinical environments ( $\mathrm{N}=11)($ Continued)

\begin{tabular}{|c|c|c|c|c|c|c|c|}
\hline De Las Cuevas C et al. [4] & 2006 & 140 & Psychiatric outpatients & $\begin{array}{l}\text { Randomized to face-to-face } \\
\text { or telepsychiatry }\end{array}$ & 24 weeks & - Clinical & $\begin{array}{l}\text { - Telepsychiatry had equivalent efficacy } \\
\text { to face-to-face care }\end{array}$ \\
\hline \multirow[t]{4}{*}{ Ruskin PE et al. [11] } & \multirow[t]{4}{*}{2004} & \multirow[t]{4}{*}{119} & \multirow[t]{4}{*}{ Veterans with depression } & \multirow{4}{*}{$\begin{array}{l}\text { Randomized to telepsychiatry } \\
\text { or in-person psychiatrist visits }\end{array}$} & \multirow[t]{4}{*}{6 months } & - Clinical & \multirow{4}{*}{$\begin{array}{l}\text { Both groups were equivalent in clinical } \\
\text { outcomes, cost, patient adherence, and } \\
\text { patient satisfaction. }\end{array}$} \\
\hline & & & & & & - Cost effectiveness & \\
\hline & & & & & & $\begin{array}{l}\text { - Healthcare resource } \\
\text { utilization }\end{array}$ & \\
\hline & & & & & & - Satisfaction & \\
\hline Bishop JE et al. [3] & 2002 & 17 & Psychiatric patients & $\begin{array}{l}\text { Randomized to } \\
\text { videoconference } \\
\text { or face-to-face }\end{array}$ & 4 months & - Satisfaction & $\begin{array}{l}\text { - Similar satisfaction observed in } \\
\text { both groups }\end{array}$ \\
\hline
\end{tabular}


Published online: 05 January 2016

\section{References}

1. Achey MA, Beck CA, Beran DB, Boyd CM, Schmidt PN, Willis AW, et al. Virtual house calls for Parkinson disease (Connect.Parkinson): study protocol for a randomized, controlled trial. Trials. 2014;15:465.

2. Ahmed SN, Mann C, Sinclair DB, Heino A, Iskiw B, Quigley D, et al. Feasibility of epilepsy follow-up care through telemedicine: a pilot study on the patient's perspective. Epilepsia. 2008;49(4):573-85.

3. Bishop JE, O'Reilly RL, Maddox K, Hutchinson LJ. Client satisfaction in a feasibility study comparing face-to-face interviews with telepsychiatry. J Telemed Telecare. 2002;8(4):217-21.

4. De Las Cuevas C, Arredondo MT, Cabrera MF, Sulzenbacher H, Meise U. Randomized clinical trial of telepsychiatry through videoconference versus face-to-face conventional psychiatric treatment. Telemed J E Health. 2006;12(3):341-50

5. Dixon RF, Stahl JE. A randomized trial of virtual visits in a general medicine practice. J Telemed Telecare. 2009;15(3):115-7.

6. Dorsey ER, Deuel LM, Voss TS, Finnigan K, George BP, Eason S, et al. Increasing access to specialty care: a pilot, randomized controlled trial of telemedicine for Parkinson's disease. Mov Disord. 2010;25(11):1652-9.

7. Ferrer-Roca O, Garcia-Nogales A, Pelaez C. The impact of telemedicine on quality of life in rural areas: the Extremadura model of specialized care delivery. Telemed J E Health. 2010;16(2):233-43.

8. Fortney JC, Pyne JM, Mouden SB, Mittal D, Hudson TJ, Schroeder GW, et al. Practice-based versus telemedicine-based collaborative care for depression in rural federally qualified health centers: a pragmatic randomized comparative effectiveness trial. Am J Psychiatry. 2013;170(4):414-25.

9. Moreno FA, Chong J, Dumbauld J, Humke M, Byreddy S. Use of standard Webcam and Internet equipment for telepsychiatry treatment of depression among underserved Hispanics. Psychiatr Serv. 2012;63(12):1213-7.

10. O'Reilly R, Bishop J, Maddox K, Hutchinson L, Fisman M, Takhar J. Is telepsychiatry equivalent to face-to-face psychiatry? Results from a randomized controlled equivalence trial. Psychiatr Serv. 2007;58(6):836-43.

11. Ruskin PE, Silver-Aylaian M, Kling MA, Reed SA, Bradham DD, Hebel JR, et al. Treatment outcomes in depression: comparison of remote treatment through telepsychiatry to in-person treatment. Am J Psychiatry. 2004;161(8):1471-6.

12. Stahl JE, Dixon RF. Acceptability and willingness to pay for primary care videoconferencing: a randomized controlled trial. J Telemed Telecare. 2010;16(3):147-51.

13. Wootton R. Twenty years of telemedicine in chronic disease management-an evidence synthesis. J Telemed Telecare. 2012;18(4):211-20.

14. Dorsey ER, Venkataraman V, Grana MJ, Bull MT, George BP, Boyd CM, et al. Randomized controlled clinical trial of "virtual house calls" for Parkinson disease. JAMA Neurol. 2013;70(5):565-70.

15. McCrossan B, Morgan G, Grant B, Sands AJ, Craig BG, Doherty NN, et al. A randomised trial of a remote home support programme for infants with major congenital heart disease. Heart. 2012;98(20):1523-8.

16. Morgan GJ, Craig B, Grant B, Sands A, Doherty N, Casey F. Home videoconferencing for patients with severe congential heart disease following discharge. Congenit Heart Dis. 2008;3(5):317-24.

17. Leon A, Caceres C, Fernandez E, Chausa P, Martin M, Codina C, et al. A new multidisciplinary home care telemedicine system to monitor stable chronic human immunodeficiency virus-infected patients: a randomized study. PLoS One. 2011:6(1):e14515.

18. Whitlock WL, Brown A, Moore K, Pavliscsak H, Dingbaum A, Lacefield D, et al. Telemedicine improved diabetic management. Mil Med. 2000;165(8):579-84.

19. Dallolio L, Menarini M, China S, Ventura M, Stainthorpe A, Soopramanien A et al. Functional and Clinical Outcomes of Telemedicine in Patients With Spinal Cord Injury. Arch Phys Med Rehabil. 2008;89(12):2332-41.

\section{Submit your next manuscript to BioMed Central and take full advantage of:}

- Convenient online submission

- Thorough peer review

- No space constraints or color figure charges

- Immediate publication on acceptance

- Inclusion in PubMed, CAS, Scopus and Google Scholar

- Research which is freely available for redistribution

Submit your manuscript at www.biomedcentral.com/submit 